\title{
Design of an Aerial Drone Delivery System
}

\author{
Aaron Don M. Africa, Robert Ianny Roi F. Quijano, Benjamin Emmanuel C. Uy \\ Department of Electronics and Communications Engineering \\ De La Salle University, Manila \\ 2401 Taft Ave., Malate, Manila 1004, \\ Philippines, aaron.africa@dlsu.edu.ph
}

\begin{abstract}
Considering the weight of the traffic in the Philippines, the delivery of products via land vehicles has long since been an inconvenience to consumers due to extensive delivery times. Due to the increasing number of people using automobiles on the road every year, traffic congestion becomes more prevalent in both rural and urban areas, especially during rush hours. To compensate for this, an alternative form of courier service via unmanned aerial vehicles (UAVs) or specific drones is proposed. Drone delivery is performed such that drones would transfer parcels from headquarters to the assigned delivery location within a maximum distance of 12 kilometers. The approximated travel time of the drones is calculated assuming perfect weather conditions, no wind turbulence, and no object collision. The estimation is made considering the average speed of the drone and the input distance from headquarters to the delivery location. A Matlab program is made that computes for the delivery time of the drone given an input between 0 to 12 kilometers. Relatively, tests show that input distances lower than 0 or greater than 12 kilometers results in an error message.
\end{abstract}

Key words: Drone, delivery, alternative, unmanned aerial vehicles (UAVs).

\section{INTRODUCTION}

Aerial drone delivery is proposed as a new alternative to the delivery system. In the Philippines, a drone delivery system has yet to be established. The reason for implementation for drone delivery comes from the need for alternatives due to problems encountered using currently established methods of delivery. When compared to land vehicles, it has even been proven that drones are much more environment-friendly than other modes of goods transportation [1]. But before actual implementation, a simulation of the system would be more beneficial [2]

So far, drone delivery is more or less in the testing phases in other parts of the world [3]. In the US, the company Amazon is conducting drone delivery service tests with their Amazon Scout platform. In Rwanda, deliveries to mountainous regions have since begun. Other countries in Europe and Asia have since followed [4].
The reason aerial drone delivery is necessary for the Philippines is due to the problems current delivery methods encounter. Current delivery methods are easily affected by traffic. Traffic congestion has been a long-fought problem faced by the Philippines. Numerous efforts have been made to circumnavigate the problem, but none have yet to prevail. Which is why introducing another method of delivery service is necessary. If the problem cannot be fixed, the solution may lie somewhere else entirely.

Drone delivery services have so far revolved around postal and goods, food delivery [5], and medical needs. This is the case as it can carry lightweight loads faster than surface vehicles during rush hours and emergencies [6]. Drone delivery for postal and goods is simply another method for delivery. But drone delivery for medical needs, on the other hand, deserves far more attention. Drone delivery for medical purposes can substantially help the people in rural or difficult to traverse regions. The people in those regions would be able to receive the things they would need to save lives. Medical needs such as blood bags would be transported much more conveniently with drones. Especially compared to the methods currently being used. To detect and identify and keep track of the drones, a possible method is to use a radio frequency-based detection system [7]. Another possible method is to use a fleet-based radar system where every drone transmits to one another [8] Due to the current limitations that battery life induces for drones, Hassija, et al, propose a charging station network system for drones [9]. Aerial base stations which could prolong the operation time of drones [10]. Routing algorithms can even be implemented to ensure the drone flight paths are optimized [11] Relatively, a fairly popular algorithm in drone technology that practitioners like to base their routing programs is the traveling salesman problem. The basic concept of the traveling salesman is that a vehicle transports drones together with the parcels and act as a temporary charging station or headquarters for the unmanned aerial vehicle (UAV) $[12,13]$. Other variations such as the truck-drone-ATV and multi-visit drone routing problem are algorithms that were based on the traveling salesman $[14,15]$. Also, routing concepts such as GeoUAVs and other obstacle avoidance algorithms are worth to note [16, 17]. Indoor delivery systems have even been developed. [18, 19, 20] Overall, multiple system implementation and optimization strategies are necessary, and efforts have been made to create such strategies $[21,22,23]$. 
So far, computational simulations have proven to be accurate with real-world data [24] Therefore the researchers of this paper seek to design a basic conceptual system for drone delivery services in the Philippines. The system would be designed with small and light packages in mind. For this system, the travel distance would be limited to the type of drone used, with package weight considered. Therefore, no specific travel distance limit would be imposed by the conceptual system design of the paper.

\section{BACKGROUND OF THE STUDY}

Drone delivery is practically in its fetal stage in the Philippines. The concept has been discussed, but the actual implementation is nearly nonexistent. The most common method of package delivery in the Philippines is through courier and delivery service. Food delivery also is done through delivery services such as GrabFood and Foodpanda. These services rely on employees with personal vehicles picking up the orders of customers and then delivering the food to them. This method of delivery also supports package delivery. While currently, this system works, the traffic situation in the Philippines causes certain dilemmas that affect delivery time, product quality, and customer satisfaction. As such, drone delivery has been considered as a possible solution to this problem [25]

A concern with drone delivery is that it may prove too costly a solution to be sustainable. Yet possibly, a field where drone delivery might prove to be relevant and necessary is in the field of healthcare. Medical supply delivery tends to be a concern in the Philippines as there tends to be a regular shortage of blood bags available for patients in need of blood transfusion in certain areas. An area with an available supply would have a hard time being able to deliver the needed item in time for the patient if conventional delivery methods were to be used. Especially considering the traffic. But with a drone, the delivery may become much easier and practical. The cost would be justified as well. In terms of the perception of drone delivery system implementation, studies show that environmental context matters [26] Considering the situation in the Philippines, drone implementation may be more than welcomed by the population. Especially if efforts are made to help the people understand the system [27].

Drone delivery has been suggested as a possible delivery system alternative across nearly all countries. Drone delivery has been successfully implemented in certain regions. Particularly, in the field of healthcare. Drone delivery has been successfully implemented in areas such as Rwanda, where medical packages are delivered to sectors in need. Drones were implemented in Rwanda due to the mountainous terrain in the region. Due to the mountainous terrain, vehicular delivery would be impractical and quite possibly hazardous. Out of necessity, drone delivery was presented as a solution. This system of delivery has so far proven successful in the region. As such, the researchers present this paper as an early study for a system that can be used to manage drone delivery systems in the Philippines.

\section{STATEMENT OF THE PROBLEM}

The implementation of unmanned aerial vehicles in logistic operations comes with three main problems in applications within urban areas. A significant problem is the finite physical capabilities of the UAVs to deliver corresponding parcels to the consumers [28] These parameters include the restricted carry capacity, variable energy consumption, low energy storage, and mediocre flight airspeed of the drone. Another apparent problem in the usage of drones for delivery services is the routing logarithm to be used to deliver products to customers $[29,30]$. The protocol should be able to address issues such as object obstructions, unfavorable weather conditions, and emergency measures in cases of unexpected errors or accidents. Consequently, the problem of security comes into play especially when transporting packages in loosely guarded places in the Philippines. There may be cases such that parcels or even the drones are stolen or obstructed by a human intervention which brings about suspicion to the fidelity and practicality of drone delivery.

\section{SIGNIFICANCE OF THE STUDY}

Traffic congestion has been a major problem in the Philippines as the number of vehicles on the roadways substantially increase over the years. Because of this, customers that afford products through delivery services suffer from long delivery times. This is mainly because parcels are delivered using surface vehicles, prevalently motorcycles. This study about drone delivery is relevant in recent times because the aforementioned problem can be resolved through means of unmanned aerial vehicles. By traveling through the airspace, such devices can relatively lessen delivery times for product outlets and significantly reduce waiting times for consumers in the Philippines. Additionally, UAVs or, specifically, drones present an opportunity to automate the process of product delivery. The concept of automated transportation of goods brings about several advantages for workers and sellers in general. Notably, drone delivery eliminates the problem of worker safety considering the road hazards when delivering through surface vehicles. Also, UAVs are low maintenance thus cost-optimal and are cost-efficient for companies when compared to the human labor force. The study of delivery drones is also significant as it can carry out a multitude of tasks that most human workers are incapable of. As an example, delivery drones can transport parcels over hard-to-reach areas such as in mountain provinces and the likes. To be direct, manual courier services can outright be replaced by drone deliveries which justifies the relevance of the study.

\section{DESCRIPTION OF THE SYSTEM}

The system to be designed will consist of a drone as the main object. The drone will be placed in the starting position. A target destination will be set, and the drone will travel to the target destination. The typical flight speed of a drone is 100 kilometers per hour. This will be considered in the program's calculations. The system will calculate the approximate time it 
Aaron Don M. Africa et al., International Journal of Emerging Trends in Engineering Research, 8(7), July 2020, 3116 - 3121

will take for the drone to reach the target destination assuming ideal weather conditions. A delivery drone is capable of carrying packages weighing between 5 kilograms to 30 kilograms at the speed of 100 kilometers per hour. A drone can travel a maximum of 24 kilometers. Meaning to and from the specified location. With that in mind, the maximum delivery distance that can be set will be 12 kilometers and the remaining 12 will be used to return to headquarters.

\section{METHODOLOGY}

For step by step procedure, the distance of the target destination will first be encoded. The program will then calculate the approximate time the drone will need to reach the target destination. The formula for travel time is the distance overrate or $\mathrm{t}=\mathrm{dr}$. Figure 1 shows the flowchart of the system.

\section{THEORETICAL CONSIDERATIONS}

This system considers obstacles as not a concern because the assumption will be that the drone will fly at an altitude above most structures. Also, the weather is assumed to be ideal because a drone would not be deployed under heavy weather conditions. Also, the assumption is that there will be multiple branches from which a drone may be deployed. Therefore, is a certain delivery destination being beyond what a drone from one branch can reach, a drone from a branch closer to the delivery destination would then be assigned to the delivery job.

\section{DATA AND RESULTS}

If the user were to input a distance lying anywhere greater than 0 kilometers and less than or equal to 12 kilometers, the system would output the approximate number of minutes the drone would need to reach the desired destination.

Figures 2 to 5 show examples of the system with various input distances:

Input distance: 4.3 kilometers

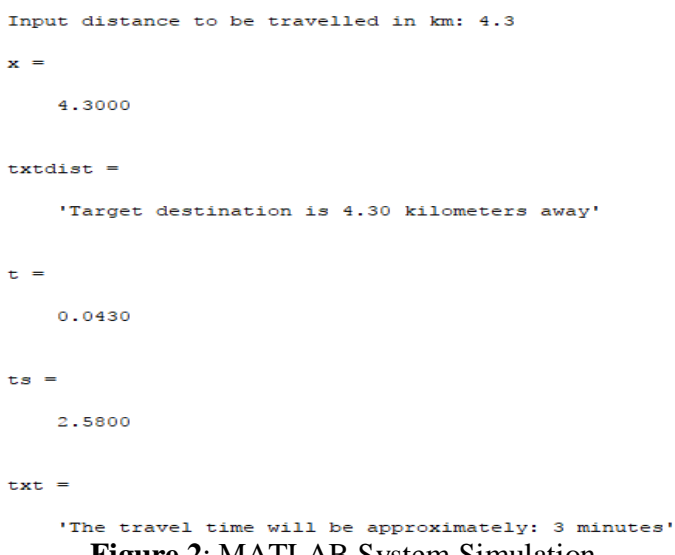

Figure 2: MATLAB System Simulation

Input distance: 8.3 kilometers

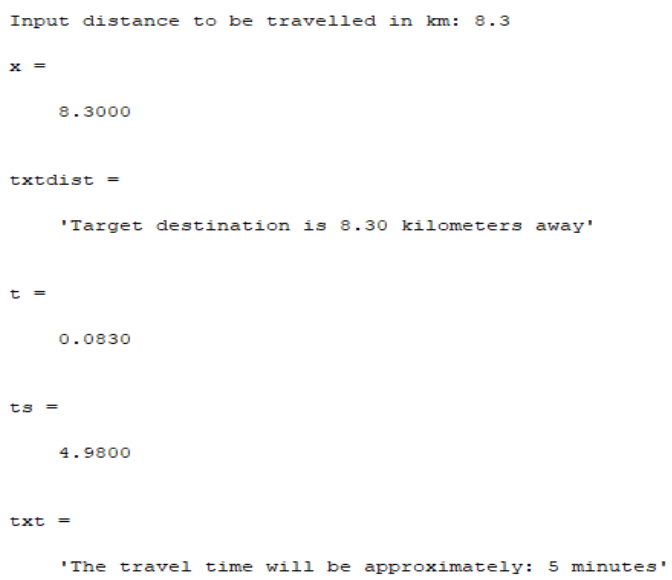

Figure 3: Pie Graph of the data 
If the user were to input a distance less than or equal to 0 or a distance greater than 12, the system would display an error message indicating that the input distance is invalid.

Input distance: 13.2 kilometers

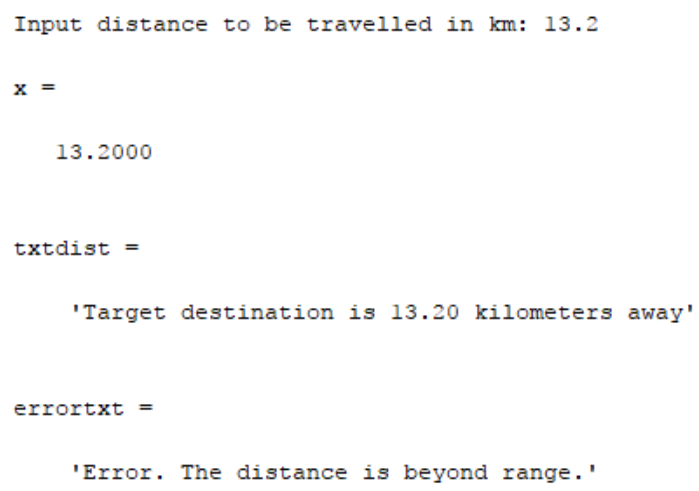

Figure 4: Input distance $13.2 \mathrm{~km}$

Input distance: -1

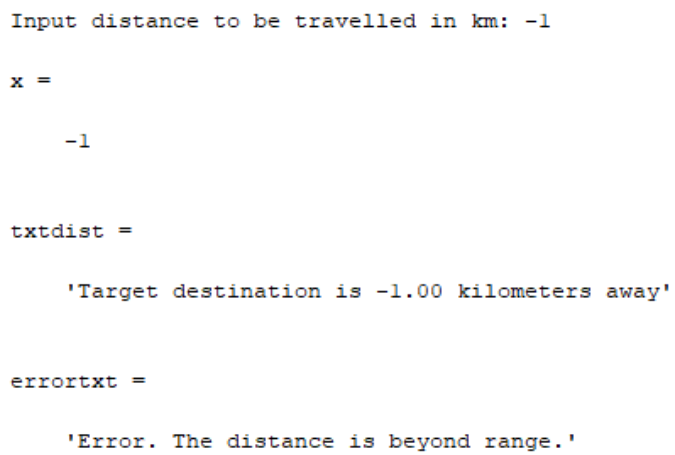

Figure 5: Input distance $-1 \mathrm{~km}$

\section{ANALYSIS OF THE DATA}

The data obtained shows how a drone delivery system could be implemented. First, the delivery destination would have to be stated. If the distance of the delivery destination is beyond the drone's capabilities, the system would reject the order and transfer it to another branch if possible.

As seen in the results presented above, the system will accept the order if the delivery destination is within 12 kilometers from headquarters. The system will approve an input distance of any value greater than 0 and less than or equal to 12 . The system will then find the approximate time it would take for the drone to reach that destination. The approximate time the drone would take is displayed in minutes. Displaying the approximate time in minutes is the most ideal because displaying the approximate time in hours would not be specific enough and displaying the approximate time in seconds would be tedious and confusing.

In the examples above, the input distances were 4.3 and 8.3 kilometers. Distances with decimals were used as examples to demonstrate that the system would consider distance minutiae in its calculations. The resulting approximate time estimates were 3 minutes and 5 minutes respectively.

Alternatively, the system would reject the order if the input were beyond the drone's capabilities. As seen above, a delivery distance of 13.2 kilometers would be rejected by the system as a delivery drone would not be able to return to headquarters if it were to travel to this distance. The system would also reject the order if the input were an impossible value such as any number less than or equal to 0 .

Therefore, the system works as a good delivery time estimator assuming certain conditions. Conditions such as the drone's travel speed being 100 kilometers per hour, and the weather conditions being ideal.

\section{CONCLUSION}

Delivery drones are becoming an actual valid method of service around the world. The drones have so far been used to transport postal packages and medical goods. Knowing this, implementing the system would create another means of delivery service in the Philippines. Certain factors necessitate the implementation of another means of delivery service in the Philippines. Namely traffic and terrain. As the primary means of delivery services in the country are vehicular, traffic plays a major role in determining the time delivery would take. In the event of an emergency delivery for medical goods, traffic would have to be ideal. A chance like that cannot be taken. To circumnavigate this, another means of delivery that doesn't have traffic conditions as a factor can be implemented. Enter a drone delivery system.

A drone delivery system can be implemented in the Philippines if the system were to be created knowing the capabilities and limitations of a delivery drone. Today's delivery drones can make a roundtrip of 24 kilometers. This means the maximum distance a drone can delivery to would behalf of that, which is 12 kilometers. The idea for the service is that there are many branches around the country from which a drone could deploy. An order would be placed, and the order's area would be input to the system. The system would then determine whether a branch could deploy a drone to deliver to that area. If the area is beyond range, the system will reject the order and pass it on to another branch. If the area is then within range of a certain branch, the system will then approve the order and deploy a drone to deliver a package. If none of the branches would be able to deploy a drone to a certain area, the system would theoretically relay the order to other means of delivery.

\section{RECOMMENDATIONS}

There are several points in this study that can be improved on concerning its complexity and flexibility. First and foremost, future endeavors may implement a program that takes into account object collision. In this study of delivery drones, the researchers took for granted that the drones would be flying above infrastructures in every delivery service. However, in certain circumstances, a drone needs to pass through obstacles 
such as electricity lines or may come into collision with living animals prevalently birds. As a result, the approximate time it takes to finish deliveries will also vary. A program that could sense such objects and recalculate the delivery time helps improve its accuracy.

Succeeding studies may also add features to the program that takes into account wind turbulence and weather conditions. Ideal conditions were assumed in the study; no such turbulence and clear weather conditions. However, such conditions are not always true in every delivery service. There may be times that tailwinds or headwinds may support or obstruct the average speed of the drone respectively; the same could be said to bad weather conditions. This would notably affect the precision of the approximated time. By factoring in wind and weather conditions in the program, the estimated time of delivery would be much more specific.

Another possible point to improve on in future research is how to compensate for the physical limitations of the drone. As stated in the study, the drone could only handle 12 kilometers, not including the return trip to the headquarters, due to restrictions in the battery life. The delivery order would then be transferred to consequent branches according to the protocol in such a case. However, there might be times when there are no branches near the assigned delivery location and is just out of coverage via drones. A plausible solution to this is the truck-drone-atv routing wherein trucks carrying the drones and its parcels go near the destination area and act as an alternative headquarters for the UAVs. Implementing this into the program, one needs to take into account the travel time of both the truck and UAV to estimate the approximate delivery time.

\section{REFERENCES}

[1] W. Chiang, Y. Li, J. Shang and T. L. Urban, "Impact of Drone Delivery on Sustainability and Cost: Realizing the UAV Potential through Vehicle Routing Optimization," Applied Energy. Vol. 242, pp. 1164-1175, 2019. https://doi.org/10.1016/j.apenergy.2019.03.117

[2] P. Bocciarelli,A. D'Ambrogio, A. Falcone, A. Garroand A. Giglio, "A Model-Driven Approach to Enable the Simulation of Complex Systems on Distributed Architectures," Simulation. Vol. 95, No. 12, pp. 1185-1211, 2019. https://doi.org/10.1177/0037549719829828

[3] A. Raj and B. Sah, "Analyzing Critical Success Factors for Implementation of Drones in the Logistics Sector using Grey-DEMATEL Based Approach," Computers and Industrial Engineering. Vol. 138, 2019. https://doi.org/10.1016/j.cie.2019.106118

[4] J. Aurambout, K. Gkoumas and B. Ciuffo, "Last Mile Delivery by Drones: An Estimation of Viable Market Potential and Access to Citizens Across European Cities," European Transport Research Review. Vol. 11, No. 1, 2019. https://doi.org/10.1186/s12544-019-0368-2

[5] J. J. Kim, and J. Hwang, "Merging the Norm Activation Model and the Theory of Planned Behavior in the
Context of Drone Food Delivery Services: Does the Level of Product Knowledge really Matter?" Journal of Hospitality and Tourism Management. Vol. 42, pp. 1-11, 2020. https://doi.org/10.1016/j.jhtm.2019.11.002

[6] D. Swanson, "A Simulation-Based Process Model for Managing Drone Deployment to Minimize Total Delivery Time," IEEE Engineering Management Review. Vol. 47, No. 3, pp. 154-167, 2019.

[7] M. F. Al-Sa'd, A. Al-Ali, A. Mohamed, T. Khattaband A. Erbad, "RF-Based Drone Detection and Identification using Deep Learning Approaches: An Initiative Towards a Large Open Source Drone Database," Future Generation Computer Systems. Vol. 100, pp. 86-97, 2019. https://doi.org/10.1016/j.future.2019.05.007

[8] J. Hao, J. Li, Y. Piand X. Fang, "A Drone Fleet-Borne SAR Model and Three-Dimensional Imaging Algorithm," IEEE Sensors Journal. Vol. 19, No. 20, pp. 9178-9186, 2019. https://doi.org/10.1109/JSEN.2019.2923529

[9] V. Hassija,V. Saxenaand V. Chamola, "Scheduling Drone Charging for Multi-Drone Network Based on Consensus Time-Stamp and Game Theory," Computer Communications. Vol. 149, pp. 51-61, 2020.

[10] Y. Özçevik and B. Canberk, "Energy Aware Endurance Framework for Mission Critical Aerial Networks," Ad Hoc Networks. Vol. 96, 2020. https://doi.org/10.1016/j.adhoc.2019.101992

[11] Y. Liu, "An Optimization-Driven Dynamic Vehicle Routing Algorithm for on-Demand Meal Delivery using Drones," Computers and Operations Research. Vol. 111, pp. 1-20, 2019.

[12] J. C. de Freitasand P. H. V. Penna, "A Variable Neighborhood Search for Flying Sidekick Traveling Salesman Problem," International Transactions in Operational Research. Vol. 27, No. 1, pp. 267-290, 2020.

[13] D. Schermer, M. Moeini and O. Wendt, "The Traveling Salesman Drone Station Location Problem," Advances in Intelligent Systems and Computing. Vol. 991, 2020. https://doi.org/10.1007/978-3-030-21803-4_111

[14] M. Moeiniand H. Salewski, "A Genetic Algorithm for Solving the Truck-Drone-ATV Routing Problem," Advances in Intelligent Systems and Computing. Vol. 991, 2020.

[15] S. Poikonenand B. Golden, "Multi-Visit Drone Routing Problem," Computers and Operations Research. Vol. $113,2020$.

[16]F. Z. Bousbaa, C. A. Kerrache, Z. Mahi, A. E. K. Tahari, N. Lagraa and M. B. Yagoubi, "GeoUAVs: A New Geocast Routing Protocol for Fleet of UAVs," Computer Communications. Vol. 149, pp. 259-269, 2020. https://doi.org/10.1016/j.comcom.2019.10.026

[17] S. Shin, Y. Kangand Y.Kim, "Reward-Driven U-Net Training for Obstacle Avoidance Drone," Expert Systems with Applications. Vol. 143, 2020.

[18] M. Kloetzer, A. Burlacu, G. Enescu, S. Caraiman and C. Mahulea, "Optimal Indoor Goods Delivery using Drones," 2019. https://doi.org/10.1109/ETFA.2019.8869278 
[19]R. Xiao, H. Du, C. Xuand W. Wang, “An Efficient Real-Time Indoor Autonomous Navigation and Path Planning System for Drones Based on RGB-D Sensor," Lecture Notes in Electrical Engineering. Vol. 586, 2020.

[20] H. Moon, J. Martinez-Carranza, T. Cieslewski, M. Faessler, D. Falanga, A. Simovic and D. Scaramuzza, "Challenges and Implemented Technologies used in Autonomous Drone Racing," Intelligent Service Robotics. Vol. 12, No. 2, pp. 137-148, 2019. https://doi.org/10.30534/ijeter/2019/13792019

[21] P. Madhuravani, K. Yamini and T. Nirmala, "Large scale community discovery of complex networks based on approximate optimization," International Journal of Emerging Trends in Engineering Research. Vol. 7, No. 9, pp. 306-310, 2019.

[22]H. Jeong,B.Song and S. Lee, "Truck-Drone Hybrid Delivery Routing: Payload-Energy Dependency and no-Fly Zones," International Journal of Production Economics. Vol. 214, pp. 220-233, 2019.

[23] A. Africa, A. Tabalan and M.Tan, "Speech emotion recognition using support vector machines," International Journal of Emerging Trends in Engineering Research. Vol.8, No. 4, pp. 1212-1216, 2020. https://doi.org/10.30534/ijeter/2020/43842020

[24] S. Wen,J. Han, Z. Ning, Y. Lan, X. Yin, J. Zhang and Y. Ge, "Numerical Analysis and Validation of Spray Distributions Disturbed by Quad-Rotor Drone Wake at Different Flight Speeds," Computers and Electronics in Agriculture. Vol. 166, 2019.

[25] J. Hwang,J.Leeand H. Kim, "Perceived Innovativeness of Drone Food Delivery Services and its Impacts on Attitude and Behavioral Intentions: The Moderating Role of Gender and Age," International Journal of Hospitality Management. Vol. 81, pp. 94-103, 2019.

[26] J.Hwang,S.Cho and W. Kim, "Consequences of Psychological Benefits of using Eco-Friendly Services in the Context of Drone Food Delivery Services," Journal of Travel and Tourism Marketing. Vol. 36, No. 7, pp. 835-846, 2019.

[27] J.Hwangand J.Choe, "Exploring Perceived Risk in Building Successful Drone Food Delivery Services," International Journal of Contemporary Hospitality Management. Vol. 31, No. 8, pp. 3249-3269, 2019. https://doi.org/10.1108/IJCHM-07-2018-0558

[28] J. Perussi,F. Gressler and R. Seleme, "Supply Chain 4.0: Autonomous Vehicles and Equipment to Meet Demand," International Journal of Supply Chain Management. Vol. 8, No. 4, pp. 33-41, 2019.

[29] H. Zhang, Z. Lei, G. Wang and J.Hwang, "Eye in the Sky: Drone-Based Object Tracking and 3D Localization," 2019. https://doi.org/10.1145/3343031.3350933

[30] S. Watkins, J. Burry, A. Mohamed, M. Marino, S. Prudden, A. Fisher, N. Kloet, T. Jakobiand R. Clothier, "Ten Questions Concerning the use of Drones in Urban Environments," Building and Environment. Vol. 167, 2020.

https://doi.org/10.1016/j.buildenv.2019.106458 